\title{
Governments Should Compel Leading Medical Journals to Correct Flaws and Mistakes Concerning COVID-19
}

\author{
Jianqing Wu, Ph.D. J.D.
}

March 31, 2020

\section{(A First Letter to U.S. Government Re: COVID-19 Catastrophe)}

The COVID-19 pandemic has gotten out of control, from a few cases to nearly 170,000 cases in the U.S. It is predicted that about $80 \%$ people will get the disease with estimated 2.2 million deaths. The world population is 7.8 billion and about 6.2 billion will get the disease. About 180 million may die from the disease. A far worse consequence is that a large number of people will suffer lung disability permanently. I will show that leading medical journals' editorial policies and review practices are primarily responsible for this disaster.

\section{A. Medicine on a Wrong Foundation}

The reason for seeing this disastrous situation is that medicine is fundamentally flawed in four core presumptions. Due to those flaws, medicine is unable to accurately evaluate specific health problems for specific persons. Those flaws were discussed in a series of articles that were posted on several preprint servers long time ago. I proved that randomized controlled trial is a wrong method for studying and developing treatment protocols: every such a protocol has no utility for specific persons. I sent a series of articles to selected Senators and House Representatives (e.g., I have asked my Representative, Anthony Brown, to relay my articles). Medical foundational flaws are reflected in the failure in the war against COVID-19.

1. Medicine provides no cure, but I found hundreds to thousands of things can be used to mitigate disease severity, based on hundreds of published studies.

2. Medicine assumes that disease severity does not depend on transmission routes, but I found direct lung exposure to the virus and later re-infection of the lung tissue are the main aggravating factors of lung damages.

3. The CDC uses a wrong default rule: infected persons wear masks and uninfected persons do not, we found that those rules are exactly wrong.

Now, this pandemic has disrupted personal life, business, social order, and all economic activities in the U.S. and the world economy. The world is run by COVID-19 until predictable cures are found.

\section{B. Leading Medical Journals Drive Medicine onto Wrong Tracks}


In addressing any medical issue, every person including governmental official, media member, and private person looks to published articles for guidance. Everyone assumes that sound, valid, and life-saving medical discoveries are published by credible medical journals. This is completely untrue. Medical journals consider a large number of trivial factors including word limitation, citation format, writing style, topic, institution affiliation, etc. Those things are reflected in their editorial policies. The merits of the medical discovery in an article is not first thing considered while the merit of an article depends on who reviews it. The bottom line is how articles affect their revenues. In resolving this question, they consider how an article affects their fee-paying clients, reactions of their experts and their existing revenue channels. If an article exposes a foundational flaw in medical research model, they do not review it but actively suppress it. The decision is NEVER based on scientific validity, but a compelling interest in financial gains. Scientific merit of medical articles may be entertained by medical journals ONLY after the articles have passed the restrictive editorial policies and arbitrary judgment by editorial members. If submitted articles challenge the foundation of medicine, appearance of such papers is equivalent to invalidating existing articles and treatments they are obligated to defend.

\section{C. "Medical Science" Is Limited to Things Medical Journals Can Profit}

My two-decade thinking allowed me to prove that all four core presumptions in medicine are incorrect when they are used in various situations, and that is precisely why no cure is found for chronic diseases and cancer.

The world has taken the merit of medical articles in a completely wrong way. Media do not cite and comment unpublished articles because media staff can make judgment only on the basis of the publication status and journal names; libraries do not index unpublished articles because they cannot make independent judgment; researchers cannot cite unpublished articles because relying on unpublished article may prevent them from getting grants (Most organizations will not fund research on concepts that have not peer-reviewed and published). Doctors cannot consider treatment protocols that have not been reviewed and published. Federal agencies cannot use unpublished articles in making policy decisions. Most people may think that only articles published by leading medical journals teach good science. Thus, editorial decisions by medical journals have the effect of determining merit for the whole world.

However, medical journals lack the ability to determine scientific merit of medical articles. To make what seems an impossible task, they use predetermined and published editorial policies for selecting articles. Those policies are written for promoting their financial interest. When such editorial policies are combined with biased internal reviewers and external reviewers, they can select only classical research articles. They all have the effect of precluding the holistic model, lifestyle-based measures, and all kinds of combination measures. They promote synthetic drugs $\mathrm{A}, \mathrm{B}, \ldots . \mathrm{N}$ and a few money-driving things as treatment options. Essentially, their editorial practices 
have built-in rejections for anything unfitted into their preferred options before the world has a chance to see, try or experience. Such a science is just an outright junk science. By decades propagation, they have convinced the world that their promoted medicine is "science" by mainly relying on flawed evidence from randomized controlled trials. They influence all fields of medicine by influencing experts, who run government agencies, manage health care industry, and influence public opinions. Under such a system, experts normally propagate medical information to further the interest of medical journals. Such concerted practice results in a large number of largely useless treatment protocols. Some treatments are outright fraud against patients: a journal publishes articles to promote them, but refuses to publish articles to prove that they actually hurt patients.

This merit-determining system prevents model flaws from being exposed and corrected. Because four key presumptions are wrong, medicine can only create treatments for controlling symptoms but not cures. They make all decisions based on present financial gain rather than potential cures. By using their editorial policies and peer reviewers, they maintain flawed research models, preclude medical discoveries, block new research directions, conceal treatment real risks, promote harmful treatment protocols in the name of "sciences". They cannot evaluate research merits because they have no such capacity. If they reject an article, they often cannot tell one good reason, this implies that they do not even understand what they review. With all those problems, their editorial policies decide which treatments to appear, who might have treatments, and who could make money in the medical industry. In the last half a year, New England Journal of Medicine, Science, Nature, and even CDC journal all have done exactly the same thing: ask me to place the article in better places (I will produce evidence in the time of my own choosing.).

When COVID-19 has turned the world upside down, medical journals continue to prevent medical discoveries from seeing the light of day. By their editorial and review practices, medicine can never find cures for cancer and chronic diseases, and researchers cannot go beyond existing medical teachings. By their bias and irresponsible practices, they are responsible for this disaster, the loss of massive number of lives, the crippled U.S. economy, and the crippled world economy.

Since medical journals' editorial policies and review practices have hurt the mankind so seriously, all governments should intervene. Medial journals should have a legal duty to get rid of junk medicine and bring real cures to the world as the minimum legal obligations. They should have firm responsibility to correct flaws and mistakes and mitigate risks for treatments they have promoted in their prior publications. Considering human resistance to changes, medical journals must make more efforts to correct flaws and mistakes than what they have contributed in creating them in the first place. Law should compel medical journals to accept completing medical models, completing medical theories, and 
use different review standards for completing medical models if peer-review should continues to exist.

From the performance of medicine in the war against COVID-19 pandemic, we can see that most medical teachings are problematic. We found that hundreds of things can be used to mitigate lung damages. However, medical journals keep publishing articles echoing the same flawed, wrong, and even fraudulent notion that no treatment is available. Medicine keeps treating all transmission routes as same and naturally is unable to teach people to block all transmission routes. Medicine could not determine benefits and risk for wearing masks by relying on flawed evidence that has been produced in flawed research model, and kept advocating wrong rules for decades. Failure to wear masks by uninfected persons in the U.S. is the main reason for losing the war against the pandemic, WHO and CDC still attempt to resolve this issue by using flawed evidence. Due to the failure of medicine and preclusion of alternative measures, the world is going to lose about 180 million lives, and the U.S. will lose from 200,000 to 2.2 million of lives. If the U.S. continues to rely on such irresponsible medical journals' merit system, Capitol Hill itself will become a battle field of the war against this and future pandemics.

It is time to completely fix medical journals' review system. I urge all lawmakers in Western nations to wear masks (see my article: https://ssrn.com/abstract=3563851). Due to suppression of my articles by medical journals, governments might have not seen my studies. It is far too late to protect all of you, but continue to rely on medical teachings can lead to much worser outcomes. Most of my articles can be found on ssrn.com and researchgate.net. 